\title{
La enseñanza por indagación en el desarrollo de habilidades científicas
}

Joaquín Alexander Sosa Solano* Doris Teresa Dávila Sanabria**

Artículo de investigación

Fecha de Recepción: 2 julio 2018.

Fecha de Aprobación: 1 noviembre 2018.

\section{Resumen}

El trabajo presentado hace parte de un proyecto de investigación en ciencias naturales, donde se presentan los aspectos teóricos y metodológicos en la implementación de la enseñanza por indagación para el desarrollo de habilidades científicas. La propuesta se plantea a partir del análisis de resultados académicos de los estudiantes, lo que condujo a una profunda reflexión acerca de la labor pedagógica de los docentes de Ciencias de la Institución y del enfoque que están dando a la educación científica. La investigación se realiza desde el enfoque cualitativo, a través de la investigación acción educativa. En los resultados de la primera fase correspondiente a la caracterización de estrategias de enseñanza utilizadas por docentes, se utilizaron como instrumentos de recolección de información, cuestionarios cualitativos y diarios de observación de clase para., las respuestas de los docentes y sus prácticas, permiten concluir que no tienen una concepción clara de la enseñanza de las ciencias, ni una estrategia definida, además de la falta de conocimiento de las habilidades científicas. En la segunda fase se implementaron las clases por indagación, utilizando como instrumentos diarios de campo y talleres, concluyendo que los estudiantes desarrollan habilidades como formular preguntas, Hipotetizar, inferir, pero se les dificulta diseñar experimentos.

Palabras claves: Educación, enseñanza, aprendizaje, ciencias naturales, motivación, indagación, habilidades científicas.

\footnotetext{
* Institución Educativa Técnica Nuestra Señora del Rosario - Boyacá Colombia alexandersosasolano@ gmail.com

** Colegio Técnico Francisco de Paula Santander - Boyacá Colombia dtdavilas@yahoo.com
}

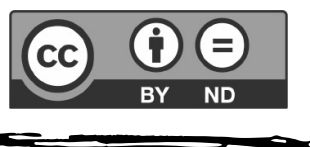




\section{Introducción}

El presente trabajo de investigación se plantea a partir del análisis de los resultados académicos de los estudiantes de la Institución Educativa Técnica Nuestra Señora del Rosario del municipio de Sativanorte Boyacá, en el área de ciencias naturales, durante el año anterior y como resultado de las reuniones del consejo académico y los comités de evaluación y promoción en la institución educativa, se evidenció que el 40 por ciento de los estudiantes de presentaban un rendimiento académico bajo en el área de ciencias naturales en comparación con los demás cursos. Igualmente, al hacer acompañamiento en aula en el grado en el año 2016, se observó que el grupo es muy participativo, se generan muchas inquietudes por parte de ellos, son curiosos, se goza de un ambiente de confianza con el maestro, pero el método de enseñanza por parte del profesor es en gran medida tradicional, siendo poco adecuado teniendo en cuenta las características de los estudiantes, donde algunos se integran poco a las actividades lo que genera indisciplina dificultando el normal desarrollo de las actividades escolares y provocando desmotivación durante la clase. Esto condujo a una profunda reflexión acerca de la labor pedagógica de los docentes de ciencias de la Institución y del enfoque que están dando a la educación científica al interior de las aulas. En este sentido, se superan las dificultades encontradas, a partir de nuevas estrategias de enseñanza.
Libâneo (1999) afirma, que en la actual dinámica de globalización se hace necesario que los maestros asuman los desafíos que exigen los avances de ciencia y tecnología, implementando estrategias didácticas que respondan a esta realidad y prepararlos para que sean capaces de aprender y así mismo, enseñar a dar solución a problemas reales. Es necesario incorporar los trabajos prácticos en la enseñanza de las ciencias a nivel de secundaria, utilizando materiales de fácil adquisición y metodologías por indagación, las cuales nacen a partir de los presaberes y la reflexión de contenidos conceptuales y procedimentales, facilitando así el desarrollo de habilidades fundamentales en el área, lo que se puede lograr a través de la transformación de los métodos de enseñanza que además de generar estas habilidades en los estudiantes mejoren el desempeño académico y permitan reflexionar sobre la manera como se viene enseñando la ciencia.

La enseñanza por indagación, ofrece a los estudiantes oportunidades para que participen activamente en el aprendizaje, hagan predicciones con sus conocimientos previos inculcados desde el hogar y en años escolares anteriores, se asombren de fenómenos naturales, formulen preguntas, hagan predicciones, diseñen experiencias para poner a pruebas sus explicaciones. Esto exige al docente un trabajo planificado, no sólo desde la transmisión de información, para que de esta forma se contribuya a mejorar los aprendizajes, aprovechar la capacidad de asombro en los estudiantes y fomentar la 
participación activa disminuyendo los focos de indisciplina y apatía que se presentan en la clase tradicional, además del desarrollo de habilidades científicas. En este sentido el propósito de esta etapa de la investigación, es hacer una reflexión acerca de las estrategias de enseñanza usadas por los docentes y su contribución al desarrollo de las habilidades científicas para a partir de este análisis proponer nuevas estrategias de enseñanza.

\section{Sustento teórico}

Garritz (2006) en la ciudad de México en su artículo "Naturaleza de la ciencia e indagación: cuestiones fundamentales para la educación científica del ciudadano" muestra cómo los estudiantes emplean la indagación para aprender los temas de la ciencia. Para lograr dichos cambios, recomendó que los profesores de ciencia utilizaran primero el laboratorio y luego, que las experiencias allí vivenciadas sirvieran como guías, más que como continuaciones, de la fase de la enseñanza teórica de las ciencias”. En este proyecto mencionan un factor fundamental para la enseñanza por indagación "el laboratorio", ya que utilizándolo de una forma correcta es una herramienta que hace posible una mejor adaptación de habilidades científicas.

Narváez (2014) en la ciudad de Palmira, Colombia en su tesis titulada "La indagación como estrategia en el desarrollo de competencias científicas, mediante la aplicación de una secuencia didáctica en el área de ciencias naturales en grado tercero de básica primaria,demostró como la estrategia por indagación, permite que los niños desarrollen habilidades propias de la indagación científica como la observación, el planteamiento de preguntas de investigación, de hipótesis y predicciones, interpretación de datos, consulta, registro de la información, entre otras. Dentro de los desempeños propios de la ciencia, los estudiantes interiorizaron la ética y la forma de pensamiento de la ciencia; así como el significado de hacer ciencias, logrando avanzar en el desarrollo del pensamiento científico. Esta tesis da una idea clara de que la enseñanza por indagación para niños de primaria permitió el desarrollo de habilidades científicas.

Así mismo, Muñoz (2014, p.56) en la ciudad de Palmira, Colombia en su tesis titulada "La indagación como estrategia para favorecer la enseñanza de las ciencias naturales" muestra un enfoque de "la indagación que se centra en el constructivismo haciendo uso del trabajo colaborativo y enfatizando el papel del estudiante como sujeto activo y responsable de su aprendizaje”. Este trabajo brinda un referente para utilizar trabajo cooperativo en las diferentes estrategias que se quieran establecer, en la medida que se propicia un clima de respeto hacia las diferencias, y se fomenta la autoestima y la motivación para generar conocimiento.

García \& Furman (2014) en la ciudad de Tunja en su artículo titulado "Categorización de Preguntas Formuladas antes y después de la
Garritz (2006) en la ciudad de México en su artículo "Naturaleza de la ciencia e indagación: cuestiones fundamentales para la educación científica del ciudadano" muestra cómo los estudiantes emplean la indagación para aprender los temas de la ciencia. 
Enseñanza por Indagación en función de los resultados obtenidos en la prueba diagnóstico", concluye que

La enseñanza como transmisión de hechos propia del modelo tradicional, como suele ser habitual en muchas instituciones incluyendo el caso de estudio, difícilmente favorece el desarrollo de habilidades científicas como la formulación de preguntas investigables, y genera que las preguntas de los alumnos apunten a conocer el significado del vocabulario científico o de datos específicos, en detrimento de preguntas que inviten a ir más allá de la información dada. (p.89)

Además estos resultados muestran que luego del desarrollo de actividades propias de la enseñanza por indagación los estudiantes desarrollaron la habilidad para formular preguntas investigables.

\section{Teorías del conocimiento}

En lo que se refiere a la enseñanza de las ciencias, Ortiz (2009) afirma que

Los docentes de ciencias naturales y educación ambiental, requieren de manera urgente la aplicación de nuevas estrategias didácticas que les permitan a los educandos acceder al conocimiento o profundizar en lo que aprenden a través de los medios de una forma interesante y productiva.(p.64)

Para ello es fundamental reconocer que el maestro no es un simple transmisor de conocimientos, sino que pasa a ejercer el papel de facilitador del aprendizaje, por lo tanto, debe capacitarse no sólo en el aspecto disciplinar, sino tener la capacidad de hacer uso adecuado de los recursos del aprendizaje y plantear técnicas y estrategias didácticas que permitan que los estudiantes participen activamente y alcancen los objetivos propuestos.

\section{Enseñanza por indagación}

En cuanto al modelo por indagación, este nace de una reacción frente al modelo de enseñanza tradicional de carácter transmisivo. Conelly y otros (1977) citado por Celis \& otros (2016),

Conciben la indagación en tres niveles, en el primer nivel la relacionan con los procesos lógicos que se usan en el desarrollo y verificación del conocimiento, en un segundo nivel la conciben como una forma o modo de aprendizaje y finalmente, la ven como una metodología de instrucción. (p.16)

Los autores hacen énfasis en la importancia de las ideas de los alumnos, en cómo los sujetos resuelven o solucionan un problema o situación, es decir, aquellos donde se formulan hipótesis, construyen conceptos o recogen datos y que además, pretenden ir más allá de la simple búsqueda de información de su objeto de estudio. Su énfasis está en desarrollar patrones de autonomía en los alumnos respecto al conocimiento científico y a la capacidad intelectual de informarse por sí mismo. En cuanto a la indagación como método de instrucción, indican que el docente debe programarle a los estudiantes preguntas que sean deíndole 
problemáticas, y una vez resueltas, invitarlos a proponer soluciones y a elaborar productos como resultado de la búsqueda de información. Se aborda además, la importancia de que los docentes involucren y capaciten a los alumnos en la necesidad de defender sus posturas argumentativas, esto mediante la discusión de los datos, aclaración de lo que expresan, firmeza en la argumentación, y desenvoltura en las conclusiones, a partir de los datos o pruebas de las fuentes consultadas.

En el contexto nacional el Instituto Colombiano para el Fomento de la Educación Superior ICFES, (2015) se define la indagación como una actividad multifacética que involucra realizar observaciones, proponer preguntas, examinar libros y otras fuentes de información, para ver que se conoce, planear investigaciones, reconocer lo que se sabe en función de nueva evidencia experimental, usar herramientas para recolectar, analizar e interpretar datos, proponer respuestas, explicaciones y predicciones, y comunicar los resultados. La indagación requiere la identificación de suposiciones, el uso del pensamiento crítico y lógico, y la consideración de aplicaciones alternativas.

\section{Habilidades científicas}

En cuanto a las habilidades científicas, se parte de la concepción de que las ciencias se constituyen como producto, entendido como el cuerpo de conocimientos y conceptualizaciones que ha sido generado a lo largo de la historia de la humanidad y como proceso, es decir, un saber hacer que incluye el conjunto de habilidades y formas de pensar mediante las cuales este conocimiento se ha construido (DeBoer, 1991).Entre las habilidades que conforman ese saber hacer de las ciencias, algunas fundamentales son la identificación de problemas, la formulación de preguntas investigables, la formulación de hipótesis y predicciones, el diseño y la realización de experimentos y con ello la observación, medición, clasificación y seriación, recolección de datos, interpretación de resultados, elaboración y comunicación de conclusiones (Arons, 1977), a través del uso de nuevas tecnologías aplicadas al desarrollo del pensmaiento científico (Davila, Galvis \& Vivas, 2015).

\section{Metodología}

El estudio se realiza desde el enfoque cualitativo que según Sampieri, Fernández y Baptista (2010), está basado en teorías constructivistas que sostienen que los individuos buscan la comprensión del mundo en el que viven. En este enfoque se confía en los puntos de vista de los participantes acerca del fenómeno que se estudia, la construcción se genera a partir de la interacción de las personas en un contexto específico y en un tiempo determinado. Así mismo, Blasco y Pérez (2007) nos dicen que lo cualitativo "estudia la realidad en su contexto natural y cómo sucede, sacando e interpretando fenómenos de acuerdo con las personas implicadas" (p.25). El estudio corresponde a investigación acción educativa, la que según Restrepo (2004) consiste en "un 
La investigación se desarrolla en la Institución Técnica Nuestra Señora del Rosario del municipio de Sativanorte que brinda atención a niños, niñas $\mathrm{y}$ adolescentes del sector urbano y rural, en los niveles de Preescolar, Básica Primaria, Secundaria y Media. proceso de reflexión y transformación continua de la práctica, para hacer de ella una actividad profesional guiada por un saber pedagógico apropiado". (p.7)

La investigación se desarrolla en la Institución Técnica Nuestra Señora del Rosario del municipio de Sativanorte que brinda atención a niños, niñas $\mathrm{y}$ adolescentes del sector urbano y rural, en los niveles de Preescolar, Básica Primaria, Secundaria y Media. La primera fase de la investigación se llevó a cabo mediante aplicación de cuestionario cualitativo dirigida a docentes, la cual fue debidamente validada y piloteada con el propósito de identificar las estrategias de enseñanza utilizadas en el área de ciencias naturales y su contribución al desarrollo de habilidades científicas y técnicas de observación con el uso de diario de observación de clases y matriz DOFA para su análisis, apoyado del registro fotográfico.

La fase de intervención tiene como propósito aplicar estrategias de enseñanza por indagación mediante talleres que involucren el desarrollo de habilidades científicas. Para ello se han planeado las clases teniendo en cuenta las habilidades que se buscan fortalecer y registrando los resultados en un diario de campo donde se analizan aspectos como: Participantes, reconstrucción del lugar del trabajo, características específicas donde se llevará a cabo la conducción de las sesiones de observación, descripción de la conducción de la clase, disposición en el espacio de los estudiantes, observaciones generales y apreciaciones personales, encuentro con la literatura existente, además de los registros fotográficos.

La población objeto de estudio corresponde a 5 docentes que trabajan el área de ciencias naturales de la institución, 3 docentes de primaria y 2 de secundaria aplicándoles un cuestionario cualitativo para determinar las estrategias de enseñanza que se utilizan en el área .

30 estudiantes, 11 niños y 19 niñas con edades entre los 13 y los 16 años de edad, pertenecientes al grado octavo de la Institución Educativa Técnica Nuestra Señora del Rosario sede urbana de carácter oficial. La población, desde el punto de vista socioeconómico, tiene las siguientes características: el 60\% corresponde al estrato uno, y el $40 \%$ restante al estrato dos; el $50 \%$ viven con ambos padres, el $30 \%$ con uno solo de los padres y un $20 \%$ de ellos viven con otra persona diferente; el 50\% de los niños presentan problemas conflictos internos y externos reflejados en el observador del estudiante y que se manifiestan en una conducta agresiva, intolerante, de baja autoestima y bajo nivel de responsabilidad académica; los estudiantes son hijos de campesinos, empleados independientes, mineros, constructores, empleadas domésticas, transportadores; según el diagnóstico realizado como parte del proyecto de dirección de curso. El ambiente en las aulas se caracteriza por permanente indisciplina lo que se puede evidenciar en el observador del alumno y por medio de la observación de conductas en las aulas. 


\section{Resultados}

Durante el año anterior y como resultado de las reuniones del consejo académico y los comités de evaluación y promoción en la Institución Educativa, se evidenció que el 40 por ciento de los estudiantes de grado séptimo presentó un rendimiento académico bajo en el área de ciencias naturales. Igualmente, al hacer acompañamiento en aula en el grado séptimo en el año 2016, se observó que el grupo es muy participativo, los estudiantes son curiosos, se generan muchas inquietudes por parte de ellos, hacen constantemente preguntas, se goza de un ambiente de confianza con el maestro, pero el método de enseñanza por parte del mismo es en gran medida tradicional, siendo poco adecuado teniendo en cuenta las características de los estudiantes, lo que genera indisciplina. Igualmente resultados de las pruebas SABER del año 2016, (Figura1) para grado noveno indican que los estudiantes en comparación con los otros establecimientos educativos tienen debilidades en la competencia de uso comprensivo del conocimiento científico, pero es muy fuerte en indagación, por eso se pretende que a través de la indagación se mejoren las demás competencias.

\section{icfes 1 OMineoucación iffes Establecimiento educativo: IE TEC NSTRA SEÑORA DEL ROSARIO C6́digo DANE: 115720000212 \\ Fecha de actualización de datos: miércoles 17 de mayo 2017}

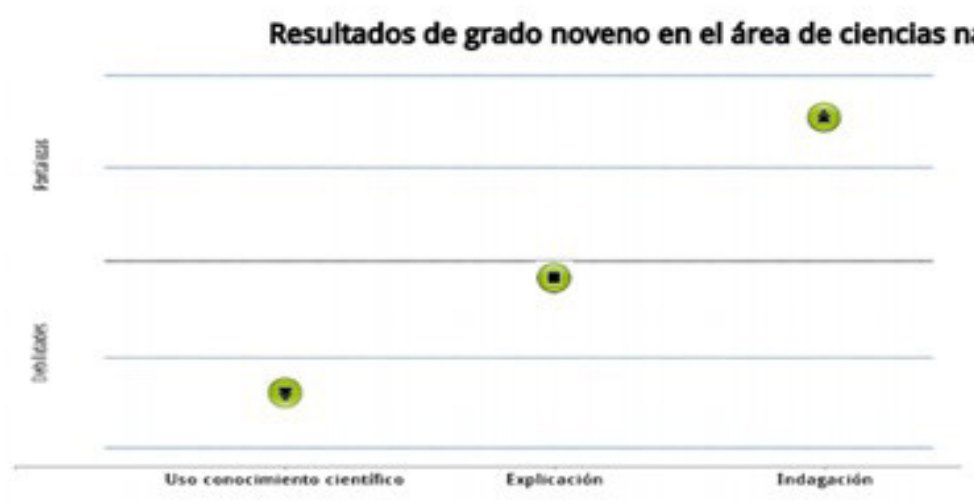

Figura 1 Resultados de las pruebas SABER año 2016 tomado de http://www2. icfesinteractivo.gov.co/ReportesSaber359/ 
El análisis de pruebas internas y externas de la institución conllevan a la revisión de los planes, pedagogía y didáctica utilizada específicamente en los grados inferiores, de tal manera que se pueda intervenir en ellos con el fin de mejorar su aprendizaje.

Primera fase Caracterización de las estrategias de enseñanza de los docentes de ciencias naturales

A continuación se muestran los resultados de la entrevista aplicada a docentes de ciencias naturales de la institución, donde se establecen diversas categorías de análisis con el objetivo de caracterizar las formas de enseñanza y su contribución al desarrollo de habilidades científicas.

\section{- Cuestionario cualitativo a docentes}

Primera categoría, enseñanza de las ciencias naturales.

En lo que se refiere a la enseñanza de las ciencias y frente a la pregunta ¿Cómo describiría el modelo de enseñanza en sus clases de ciencias naturales? los docentes respondieron: "La enseñanza a partir de la manipulación y el contacto con el entorno"; "Activo vivencial, utilización de libros, guías, tomando como punto de partida la realidad"; "Paseos o salidas (diario de campo)"; "Modelo de recepción con significados, apoyado por las TIC".

Frente a la pregunta ¿Cuáles son o han sido las principales dificultades que se le han presentado como docente, en la enseñanza de las ciencias naturales? Los docentes respondieron: "La falta de recursos y poco interés de algunos estudiantes"; "Falta de material didáctico $y$ de conciencia en mejorar nuestro entorno"; "Muchos estudiantes pequeños y algunos juegan mientras otros observan $y$ trabajan"; "Bajas expectativas por parte de un porcentaje de los estudiantes $y$ en ocasiones, bajo nivel de matemáticas, las cuales son esenciales para el desarrollo del área". En las respuestas se puede evidenciar que una de las dificultades más notorias es el poco interés por parte de los estudiantes hacia las ciencias provocando distracciones. En este sentido Garritz (2006) afirma, que la desmotivación proviene por el uso del enfoque tradicional de enseñanza de la ciencia, que al ser expositiva, promueve una visión de las ciencias naturales como un conjunto de datos a ser memorizados.

Frente a la pregunta ¿Cómo considera que aprenden los alumnos las ciencias naturales? los docentes respondieron: "A través de la observación"; "A través de la observación y la manipulación de elementos"; "Cuando se parte de una realidad demostrando con bechos lo que sucede"; "Más práctica que teoría, sin embargo, los estudiantes no dan la importancia debida a la parte teórica y se limitan a copiar y pegar sin el respectivo análisis". En las respuestas se puede evidenciar que los docentes piensan que la manera en que los estudiantes aprenden las ciencias naturales es a través de la observación y las prácticas de laboratorio, estrategias que promueven algunas de las habilidades científicas, más sin embargo, al hacer observación en el aula se evidencia que en su modelo de enseñanza no lo aplican. 
Frente a la pregunta ¿Qué dificultades presentan los estudiantes con el uso de esa metodología? Los docentes respondieron "Todos quieren participar cuando el tema se entiende desde un principio $y$ se torna interesante, pero se genera indisciplina"; "Que algunos no cuentan con los materiales suficientes para realizar la observación"; "distracción"; "Los que tienen bajo interés se desmotivan aún más". En las respuestas se puede concluir, que no existe el hábito del trabajo práctico, que existe desorganización por parte del maestro, falta motivación para la consecución de los materiales, para los docentes es más fácil tenerlos quietos, que haciendo una actividad que genere interés ya que temen perder el control de la disciplina.

Frente a la pregunta ¿Por qué motivos cree usted que a muchos estudiantes se les dificulta el aprendizaje de las ciencias naturales? Los docentes respondieron: "Por la falta de contacto con la naturaleza"; "Falta de interés; "falta de recursos"; "Porque el docente no logra captar desde un principio la atención, falta de motivación". De las respuestas se puede inferir la falta de motivación, que bien podría presentarse por la valoración negativa y el desinterés de los estudiantes hacia la ciencia, sumado a la enseñanza tradicional. Autores como Yager \& Penick, (1986); Solbes \& Vilches, (1997); Fensham, (2004) atribuyen este desinterés básicamente a los contenidos y la metodología de la enseñanza, unido al rechazo a las innovaciones propuestas por la didáctica de las ciencias, donde como lo afirman Solbes, Montserrat \& Furió (2007) es atribuido, por los propios docentes, a dos causas: Un currículo educativo amplio con un número reducido de horas lectivas y falta de formación en didáctica de las ciencias, por último, otra causa es el carácter tradicional de la evaluación del aprendizaje de las ciencias.

\section{Segunda categoría, uso de las TIC en el área de ciencias.}

En lo que se refiere al uso de las Tecnologías de la Información y la Comunicación (TIC) se presentan los siguientes resultados:

Frente a la pregunta ¿Utiliza las TIC como estrategias de enseñanza en el área de ciencias naturales? Los docentes respondieron en su mayoría "sí", frente a la pregunta: Señale el tipo de ayudas TIC, qué usted utiliza para la enseñanza de las ciencias los docentes respondieron: "video beam"; "cámara fotográfica"; "no se cuenta con un salón asignado"; "ninguno, no se cuenta con el material suficiente"; "Computadores portátiles, video beam, videos, internet, cámara fotográfica”. Se encuentra en las respuestas que los docentes en primera medida no conocen los recursos existentes y aunque usan una serie de herramientas tecnológicas, no evidencian en sus respuestas que tenga un objetivo claro hacia que habilidad o habilidades científicas se puede dirigir una actividad pedagógica. Autores como Maggio (2012) y Tortosa (2012) coinciden en afirmar que la inclusión de las TIC en las prácticas de enseñanza aporta nuevas oportunidades para construir entornos de aprendizaje más significativos para los estudiantes.
"Todos quieren participar cuando el tema se entiende desde un principio y se torna interesante, pero se genera indisciplina"; "Que algunos no cuentan con los materiales suficientes para realizar la observación"; "distracción"; "Los que tienen bajo interés se desmotivan aún más”. 
Tercera categoría: Desarrollo de habilidades científicas en las clases de ciencias.

En lo que se refiere a las habilidades científicas se presentan los siguientes resultados: Frente a la pregunta ¿Conoce usted que son las habilidades científicas? Todos los docentes respondieron "si". Frente a la pregunta, si su respuesta fue afirmativa explique cuáles son, respondieron: "Son actitudes que tiene el ser bumano, para observar, explorar, organizar y analizar, hechos, fenómenos"; "Las facultades de investigar, el uso de las TIC"; "Es la motivación que existe en la persona por averiguar, indagar y crear cosas para beneficio del entorno, que sea por causa benéfica para todo ser perteneciente a la naturaleza"."Son cinco: Identificar, Indagar, Explicar, Comunicar y Trabajo en equipo (todas ellas parten desde un problema específico o una inquietud simple - como en el área de Tecnología)". Según la literatura estudiada, las respuestas permiten determinar que los docentes en su mayoría no conocen que son las habilidades científicas. En este sentido si no existe una conceptualización clara, no hay un sentido pedagógico hacia este fin.

Frente a la pregunta ¿Considera que las estrategias que usted utiliza en la enseñanza de las ciencias naturales son las adecuadas para desarrollar habilidades científicas? ¿Por qué? Los docentes respondieron: "Si son las adecuadas, en lo posible siempre recuerdo que bay un porque para que, como, cuándo, dónde"; "Las TIC, porque enseñan a educar científicamente y a desarrollar sus babilidades de investigación"; "Si porque el niño tiene la posibilidad de observar y analizar fenómenos naturales, vivencias de seres vivos en el entorno"; "En ocasiones no se aplican dinámicas grupales lúdicas para vencer la monotonía de la teoría y se espera que todos los estudiantes sean responsables de las lecturas recomendadas, las cuales, en ocasiones no ocurren."

Frente a la pregunta. Mencione alguna experiencia realizada que considere que ha permitido mayores logros en el aprendizaje de los estudiantes con respecto a las ciencias naturales, a este respecto los docentes respondieron: "Reconocimiento de seres vivos y seres no vivos en el entorno"; "con los estudiantes analizandolosestadosdela materia, seusaron muchos recursos del medio y permitió que el estudiante mejorara sus conocimientos"; "son algunas que ban becho pensar a los estudiantes y en el momento reaccionan a algunas de sus irresponsabilidades pero luego las olvidan con respecto al cuidado del medio ambiente para conservar un mejor vivir"'CConstrucción de una máquina de Goldberg (2014), la cual sirvió para enseñar al menos 8 temas del Área de Física". Según las respuestas anteriores se puede concluir que cuando trabajan con material real hay más motivación, igualmente al construir artefactos y al efectuar salidas pero no hay claridad sobre objetivos de aprendizaje.

\section{- Observación de clase y análisis} DOFA.

A continuación se presentan los resultados de la observación de clases de ciencias naturales de primaria, secundaria y media. Como instrumento de recolección de la información se usó diario de campo abordando las mismas 
categorías de análisis de las entrevistas. En el diario de campo se estableció el grado, la asignatura, características de los estudiantes, perfil del docente, de la misma forma se establecieron las categorías de análisis: Estrategias de enseñanza, habilidades científicas y uso de las TIC. Los datos obtenidos fueron analizados a través de matriz DOFA. (Ver tabla 1)

Tabla numero 1

Matriz DOFA

\begin{tabular}{|c|c|}
\hline \multicolumn{2}{|c|}{ Matriz DOFA } \\
\hline $\begin{array}{l}\text { Fortalezas: } \\
\text { Existe una buena relación estudiante - } \\
\text { maestro en todos los casos. Los docentes } \\
\text { tienen el material necesario para la clase, } \\
\text { son claros con el objetivo de la clase. } \\
\text { Los docentes favorecen el trabajo } \\
\text { cooperativo y colaborativo, tienen los } \\
\text { conocimientos disciplinares para el } \\
\text { desarrollo de la clase. } \\
\text { De los cinco docentes, uno es licenciado } \\
\text { en ciencias naturales, uno es licenciado } \\
\text { en física, los otros docentes son } \\
\text { licenciados en Básica Primaria. } \\
\text { El docente de ciencias naturales de } \\
\text { grado séptimo y el de grado décimo } \\
\text { en primera medida indagan sobre } \\
\text { conceptos previos en los estudiantes y } \\
\text { explica la competencia a fortalecer. }\end{array}$ & $\begin{array}{l}\text { Debilidades: } \\
\text { Los docentes trabajan a partir de } \\
\text { objetivos de enseñanza sin tener en } \\
\text { cuenta los objetivos de aprendizaje, } \\
\text { las clases se efectúan bajo el enfoque } \\
\text { tradicional de la enseñanza, no hay } \\
\text { construcción de conocimiento, es } \\
\text { el maestro quien da los conceptos } \\
\text { y suministra en gran parte la } \\
\text { información, los estudiantes } \\
\text { responden a preguntas establecidas } \\
\text { previamente por el docente, no } \\
\text { se da espacio para que formulen } \\
\text { preguntas, ni se permite durante la } \\
\text { clase que ellos busquen información } \\
\text { por otros medios. } \\
\text { Existe poca formación en didáctica } \\
\text { de las ciencias, en especial en } \\
\text { docentes de primaria. Los docentes } \\
\text { conocen los temas pero no usan } \\
\text { estrategias didácticas innovadoras } \\
\text { y se observa baja motivación por } \\
\text { el aprendizaje. Algunos estudiantes } \\
\text { no participan. No hay evidencia de } \\
\text { desarrollo de habilidades científicas. }\end{array}$ \\
\hline
\end{tabular}




\section{Oportunidades:}

Existe voluntad de los docentes por aprender nuevas estrategias de enseñanza. Los estudiantes son curiosos, preguntan constantemente, lo que puede facilitar el uso de estrategias de enseñanza por indagación. La institución cuenta con suficientes recursos para el apoyo a los procesos de enseñanza, además de los recursos del medio. Existe una economía y situación del entorno clara que reconocen los estudiantes y sobre la cual se pueden trabajar muchas habilidades científicas desde el componente ciencia tecnología sociedad y ambiente.

\section{Estrategias y acciones FO:}

- Estrategias de enseñanza: Existiendo por parte del maestro buen dominio curricular, se facilita la búsqueda de estrategias didácticas que permitan mejores resultados en el desempeño docente. Estudiantes activos que favorecen el trabajo en equipo y que pueden contribuir exitosamente al proyecto. El interés en el video y en las imágenes proyectadas en la clase de grado séptimo, constituye un diagnóstico adecuado que demuestra la pertinencia del uso de TIC en el desarrollo de habilidades científicas.

Habilidades Científicas: Existe gran capacidad de asombro en los estudiantes, preguntan frecuentemente, el docente brinda confianza, lo que se puede aprovechar para fortalecer habilidades, motivación permanente e interés cuando se les presenta algo nuevo, las características de los estudiantes permiten deducir que el docente debe apoyarse en los estudiantes para el desarrollo de las estrategias pedagógicas y didácticas. Sería importante asignar un salón para el docente que pueda organizar como aula especializada de ciencias naturales con el propósito que sirva de motivación para los procesos de enseñanza aprendizaje. Uso de recursos de la institución y el traído por estudiantes. Se deben favorecer diversos tipos de trabajo de roles (trabajo cooperativo o colaborativo).

Uso de Herramientas TIC: En la clase donde se utilizó como recurso un video se observó gran motivación y generó preguntas en los estudiantes, lo que permite determinar que el uso de herramientas TIC pueden ser una contribución importante para el desarrollo de habilidades científicas. 


\section{Estrategias y Acciones DO}

Estrategias de enseñanza: Buen dominio curricular, que facilita la implementación de la estrategia para buscar fomentar habilidades científicas, además de buscar estrategias didácticas que permitan mejores resultados en el desempeño docente. Los estudiantes al principio muestran indisciplina, estudiantes tienen buena disposición al trabajo en equipo lo que favorecerá el uso de trabajo a partir de la indagación para desarrollar en ellos habilidades científicas. Estudiantes muy concentrados y atentos que pueden en un momento ser apoyo para la organización de la clase. La curiosidad y capacidad de asombro puede constituirse en un detonante para fortalecer la participación de los estudiantes. Aunque no existe una didáctica adecuada al estilo de los estudiantes, la buena disposición de los docentes permite concluir que la presente investigación será de provecho para toda la institución. Los docentes a pesar de las dificultades en la enseñanza, son profesionales idóneos con deseo de aprender y que pueden mejorar sus prácticas a partir del análisis de las dificultades presentadas en el desarrollo de sus clases y tomar provecho de las capacidades de los estudiantes para mejorar la calidad educativa. Cada sesión de clase debe tener una planeación de clase y de ser necesario una guía de clase donde se incluya entre otros, estándares, objetivo, habilidad o habilidades científicas a potenciar, materiales, metodología, rejillas o rúbricas de evaluación, que el estudiante desde el inicio de su clase debe conocer. Las características propias del contexto escolar y el contexto municipal, favorecen la enseñanza por indagación, competencia que se constituye en fortaleza en las pruebas saber.

- Habilidades científicas: Ante el hecho de que la enseñanza tradicional causa desmotivación y aburrimiento en los estudiantes, se reflexiona sobre al uso de estrategias por indagación generando el gusto por las ciencias naturales. Cuando se desenvuelven en un marco escolar indagatorio los estudiantes aprenden habilidades de investigación científica que desarrollan y utilizan, su proceso de aprendizaje implica recolectar pruebas, elegir las fuentes de información y utilizarlas adecuadamente, y mantener una discusión abierta y permanente acerca de todos los puntos de sus indagaciones. La comprensión de los procesos y contenidos de la ciencia se facilitará si los estudiantes se involucran profundamente en su propio aprendizaje, si se toman en cuenta sus opiniones y elecciones, sus experiencias previas e ideas preexistentes y se tratan temas que les resultan interesantes, relacionados con su vida diaria, sus inquietudes y la vida de su comunidad. Esto ayudará, además, a que desarrollen actitudes positivas y constructivas hacia la ciencia y el aprendizaje de la ciencia.

Uso de herramientas TIC: Algunos docentes tiene poca formación en el uso de las TIC, y por ende no las usan en sus clases. La motivación de los estudiantes ante este recurso motivará a los docentes a su uso con propósito pedagógico. 
Finalmente, se puede evidenciar que los docentes utilizan como modelo de enseñanza la tradicionalista que según Flórez \& Tobón (2001), se da desde el enfoque academicista, verbalista, bajo un régimen de disciplina a estudiantes que son básicamente receptores, Canfux (1996) asegura, que el profesor generalmente exige del alumno la memorización de la información que narra y expone

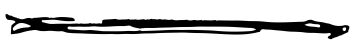

\section{Estrategias y Acciones FA:}

La formación del docente y el proceso de investigación a través de un buen diagnóstico permitirán mitigar la amenaza del contexto escolar y cultural de los estudiantes, tomado este como fortaleza y oportunidad de trabajo en el aula, igualmente las estrategias desde la indagación permiten apoyar a estudiantes con dificultades.

El análisis permite afirmar que la intención del docente en la enseñanza no corresponde al interés del estudiante, a pesar de que los docentes aseguran necesaria más la práctica que la teoría, prevalece la teoría a partir de los conceptos dados por el docente. Se observa que el uso de las TIC, es del agrado de los estudiantes, lo que permite concluir que al promover la accesibilidad de los estudiantes al mundo digital, se ayuda a contextualizar el conocimiento.

Finalmente, se puede evidenciar que los docentes utilizan como modelo de enseñanza la tradicionalista que según Flórez \& Tobón (2001), se da desde el enfoque academicista, verbalista, bajo un régimen de disciplina a estudiantes que son básicamente receptores, Canfux (1996) asegura, que el profesor generalmente exige del alumno la memorización de la información que narra y expone, refiriéndose a la realidad como algo estático y detenido, siendo en ocasiones la disertación completamente ajena a la experiencia existencial de los alumnos y los contenidos se ofrecen como segmentos de la realidad, desvinculados de su totalidad.

En cuanto a las habilidades científicas, aunque en la entrevista aseguran conocerlas y algunos docentes enumeran algunas de ellas, no se fortalecen en la clase. Scheuer, Pérez, Mateos \& Pozo (2006, p.34), explican que existe una naturaleza implícita e intuitiva de las representaciones de los maestros sobre la enseñanza y el aprendizaje y distinguen entre representaciones implícitas, que son un saber hacer (correspondería a las prácticas educativas de los maestros), de las representaciones explícitas o declarativas, que corresponde a lo que los maestros dicen sobre cómo enseñan y aprenden los alumnos. Briscoe (1991) asegura, que existen discrepancias importantes entre lo que los docentes expresan y su comportamiento observado en clase. Iturralde \& Rocha (2006) afirman, que las concepciones de los docentes sobre la ciencia, su contenido y didáctica, permiten pensar cómo intervenir en la formación del conocimiento y la enseñanza, por lo que es necesario indagar lo que el docente concibe como enseñanza y cómo actúa profesionalmente.

\section{Segunda fase, clases por indagación}

En esta fase se dan a conocer los resultados del proceso de intervención, al aplicar 5 clases por enseñanza con indagación en el grado octavo, teniendo en cuenta aspectos como: desarrollo de habilidades 
científica mediante planeación de clase, aplicación de talleres indagatorios y diarios de campo describiendo el desempeño de los estudiantes para cada sesión. Planificar una clase de enseñanza por indagación implica anticipar, prever, organizar y decidir cursos de acción. Es importante organizar de manera coherente lo que se quiere lograr con los estudiantes en el aula, lo que implica tomar decisiones previas a la práctica sobre qué es lo que se aprenderá, para qué se hará y cómo se puede lograr de la mejor manera. En la planeación se tuvieron en cuenta las siguientes etapas: focalización, exploración, reflexión y evaluación, utilizando trabajo colaborativo entre los estudiantes. En lo que se refiere a habilidades científicas se utilizaron el formulas preguntas, Hipotetizar, inferir y diseñar experimentos. A continuación el resultado del desarrollo de habilidades científicas mediante la enseñanza por indagación. (Ver tabla 2).

Tabla 2 habilidades científicas trabajadas en enseñanza por indagación.

\begin{tabular}{|c|c|c|}
\hline Habilidades científicas & Resultado & Contraste teórico \\
\hline $\begin{array}{l}\text { Formular preguntas } \\
\text { investigables }\end{array}$ & $\begin{array}{l}\text { Si hay desarrollo de esta habilidad } \\
\text { ya que se observa que } 4 \text { de los } \\
5 \text { grupos de los estudiantes } \\
\text { formulan preguntas que pueden ser } \\
\text { investigables. }\end{array}$ & $\begin{array}{l}\text { Una de las habilidades fundamentales de los } \\
\text { estudiantes para realizar indagación científica } \\
\text { es la de identificar cuestiones que puedan } \\
\text { responderse por medio de investigaciones } \\
\text { (Llewellynd 2005) }\end{array}$ \\
\hline Hipotetizar & $\begin{array}{l}\text { Si hay desarrollo de esta habilidad } \\
\text { ya que se observa que } 4 \text { de los } 5 \\
\text { grupos de los estudiantes logran } \\
\text { formular hipótesis. }\end{array}$ & $\begin{array}{l}\text { Gómez \& Pérez (2013) nos dicen que la } \\
\text { indagación es un modo de aprendizaje y } \\
\text { metodología de la instrucción, por el cual se } \\
\text { debe hacer énfasis en la curiosidad del alumno } \\
\text { como los actores que resuelven o solucionan un } \\
\text { problema o situación concreta, de manera que } \\
\text { los estudiantes formulan hipótesis a partir de sus } \\
\text { preguntas, estas deben ser capaces de comprobar. }\end{array}$ \\
\hline Diseñar experimentos & $\begin{array}{l}\text { No hay desarrollo de esta habilidad, } \\
\text { los resultados revelan que la mayor } \\
\text { parte de los alumnos no fue capaz } \\
\text { de diseñar un experimento, } 1 \text { grupo } \\
\text { de trabajo diseñó un laboratorio en } \\
\text { el cual no daban ninguna respuesta, } \\
2 \text { grupos de trabajo elaboraron una } \\
\text { comparación muy elemental que } \\
\text { daban respuesta pero no miraban } \\
\text { las variables. Solo un grupo de } \\
\text { trabajo planteó una comparación } \\
\text { y una posible medición para poder } \\
\text { establecer el resultado en donde } \\
\text { ya formulaban y ningún grupo } \\
\text { mostró la habilidad de diseñar un } \\
\text { experimento de forma completa }\end{array}$ & $\begin{array}{l}\text { Di Mauro \& Furman (2014) se explica muy bien } \\
\text { cómo medir el nivel en el que los estudiantes } \\
\text { adquieren la habilidad del diseño experimental, } \\
\text { ellas analizaron las respuestas de los alumnos y se } \\
\text { estableció una escala en función de las habilidades } \\
\text { de diseño experimental. Se plantearon } 4 \text { niveles, } \\
\text { nivel 1: ausente, nivel 2: incipiente, nivel 3: en } \\
\text { desarrollo y nivel 4completo. }\end{array}$ \\
\hline
\end{tabular}




\begin{tabular}{|c|c|c|}
\hline Inferir & $\begin{array}{l}\text { Si hay desarrollo de esta habilidad, } \\
\text { con las respuestas de los estudiantes } \\
\text { se puede describir que infirieron } \\
\text { muy bien ya que interpretaron } \\
\text { un fenómeno en base a una o } \\
\text { varias observaciones al realizar } \\
\text { un experimento analizando } \\
\text { detalladamente cada proceso de } \\
\text { este, y todos los grupos infirieron } \\
\text { de una forma correcta. }\end{array}$ & $\begin{array}{l}\text { Según Merino dela Fuente(2007) es recomendable } \\
\text { para finalizar enseñanza de las ciencias los } \\
\text { enfoques deductivos, los cuales ayudan al } \\
\text { alumnado a su capacidad de abstracción, y es } \\
\text { conveniente que proceso experimental se realice } \\
\text { en un contexto flexible, crítico y cooperativo, que } \\
\text { invite al análisis y a la confrontación de hipótesis } \\
\text { y que favorezca el debate, la comunicación, las } \\
\text { ideas intuitivas del alumnado. }\end{array}$ \\
\hline Predecir & $\begin{array}{l}\text { Si hay desarrollo de esta habilidad, } \\
\text { los } 5 \text { grupos de trabajo predijeron } \\
\text { de una forma correcta lo que } \\
\text { iba a pasar antes de realizar un } \\
\text { experimento. }\end{array}$ & $\begin{array}{l}\text { Según Arencibia \& otros (2006) se considera } \\
\text { buen dominio de la habilidad de predecir si } \\
\text { el alumno logró interpretar adecuadamente el } \\
\text { fenómeno y además tuvo en cuenta para ello las } \\
\text { condiciones en que tuvo lugar y la precisión de } \\
\text { los datos técnicos y experimentales, aś como las } \\
\text { leyes del desarrollo para su solución y si hiciese } \\
\text { la inferencia de las consecuencias de los hechos. }\end{array}$ \\
\hline
\end{tabular}

Se describe que mediante la indagación los estudiantes guiados por el docente tienen la capacidad de formular preguntas investigables, por lo tanto, el planteamiento de preguntas, especialmente aquellas preguntas investigables, que son las preguntas que puedan ser respondidas a través de experimentos, mediciones u observaciones, es un aprendizaje clave en las clases de Ciencias Naturales. La hipótesis de los estudiantes en este caso guía y orienta en lo que se pretende buscar o se trata de comprobar, es una probable respuesta al interrogante de la pregunta de investigación, siendo un resultado importante de la indagación. El inferir es una habilidad que los estudiantes desarrollan muy bien por la indagación haciendo que ellos analicen de una forma correcta lo observado. Con respecto a predecir los estudiantes pueden llegar a concluir de una forma acertada resultados de experimentos antes de que estos los realicen. Por último se puede describir que el diseño de experimentos es una habilidad que se les dificulta adquirir a los estudiantes tanto de primaria como de bachillerato, posiblemente por enseñanza que han recibido anteriormente donde el docente les da la respuesta a todo, no dejando que el estudiante busque por si solos esas respuestas, lo que hace necesario programar más actividades en donde se apliquen estrategias para desarrollar esta habilidad.

\section{Conclusiones}

Los docentes no tienen una concepción clara de la enseñanza de las ciencias, ni una estrategia definida, además de la falta de conocimiento de las habilidades científicas, lo que justifica el desarrollo de estas habilidades a través de la transformación de los métodos de enseñanza. El reflexionar sobre la manera como se viene enseñando las ciencias naturales constituye una oportunidad de 
entender que en la medida en que el mundo exterior del estudiante sea más rico, más interesante y cercano a sus posibilidades de interacción, podrá acercarse al conocimiento, y comprender su contexto social.

Existe una valoración negativa y un desinterés del alumnado hacia la ciencia que es causado por la ausencia de valor a la educación científica, la enseñanza tradicional y la consideración de las mismas que se tiene en el sistema educativo.

Frente al enfoque de la gestión del conocimiento científico, los docentes y estudiantes, deben ser competentes en la utilización y el desarrollo de tecnologías para el desarrollo eficiente de las habilidades científicas, para que a partir de una cantidad de datos se busque en los procesos de enseñanza motivar la posibilidad de analizarlos y construir conocimiento científico.

Los docentes tiene la obligación de promover la indagación en los estudiantes para de esta forma aprender habilidades de investigación científica que puedan utilizar. La comprensión de los procesos y contenidos de la ciencia se facilitará si los estudiantes se involucran profundamente en su propio aprendizaje, si se toman en cuenta sus opiniones y elecciones, sus experiencias previas, si se buscan temas de su interés relacionados con su vida diaria, sus inquietudes y la vida de su comunidad. Esto contribuirá a que desarrollen actitudes positivas y constructivas hacia la ciencia y el aprendizaje de la ciencia.

Se evidencia que una buena planificación y guía por parte del docente ayuda a promover a los estudiantes la construcción de sus propios conocimientos reestructurando los conocimientos previos, para formar nuevos.

La enseñanza por indagación es una metodología en la que se puede desarrollar habilidades científicas como planteamiento de preguntas investigables, planteamiento de hipótesis, inferir y predecir, lo que se hace indispensable tener una buena planeación para lograr ese objetivo.

Es necesario trabajar más en lo que se refiere al diseño de experimentos ya que los estudiantes presentaron muchas dificultades con respecto a esta habilidad científica, esto debido en gran medida a las estrategias tradicionales utilizadas por los docentes de ciencias, en donde no se fortalece las capacidades de este por analizar sino solamente por memorizar.

\section{Limitaciones del estudio}

Poca información bibliográfica con respecto a las habilidades científicas en los estudiantes.

Poco tiempo para desarrollar el proyecto.

\section{Prospectiva}

Utilizar las tics como estrategia para una clase por enseñanza por indagación. 


\section{Referencias}

Arencibia \& otros (2006). Evaluación y desarrollo de las habilidades Interpretar y Predecir en Fisiología I en los estudiantes de Medicina. La Habana. Cuba

Arons, A.B. (1977). The various languages. New York: Oxford University Press.

Aviles, G. (2011). La metodología indagatoria: una mirada hacia el aprendizaje significativo desde "Charpack y Vygotsky". Intersedes: Revista de las Sedes Regionales, Costa Rica, 12. (13), p.133-144.

Bertelle, A., Iturralde, C., \& Rocha, A. (2006). Análisis de la práctica de un docente de Ciencias Naturales. Universidad Nacional del Centro de la Provincia de Buenos Aires, Argentina. Revista Iberoamericana de Educación. https://doi. org/10.35362/rie3742698

Blasco, E., \& Pérez, A. (2007). Metodologías de investigación en las ciencias de la actividad física y el deporte: ampliando horizontes. Editorial Club Universitario. España.

Briscoe, C. (1991). The Dynamic Interactions Among Beliefs, Role Metaphors and Teaching Practices: A Case Study of Teacher Change. Science Education, 75, pp. 185-199.

Canfux, V. (1996). Tendencias pedagógicas contemporáneas. Ibagué: Corporación Universitaria de Ibagué. Cárdenas, A. (2001).

Casilimas, C. A. (2002). Investigación cualitativa. Bogotá, Colombia: ARFO editores e impresores.

Celis \& otros (2016). la técnica de indagación "deylu" y su influencia en la mejora de la actitud científica en el área de ciencia y ambiente en los niños de $2^{\circ}$ de educación primaria del colegio "nuevo peru” urb. Trujillo. Perú.

Connelly, F.M., Finegold, M., Clipsham j. \& Wahlstrom, M. (1977). Scientific Enquiry and the Teaching of Science. The Ontario Institute for Studies in Education.

Dávila, D., Galvis, A., \& Vivas, R. (2015). Sitio Web como estrategia de enseñanza en la educación para la sostenibilidad. Praxis « Saber, 6(11), 115 - 138. https://doi.org/10.19053/22160159.3577

Deboer, G.E. (1991). A History of Ideas in Science Education: Implications for Practice. Nueva York: Teachers College Press.

Di mauro M \& Furman M. (2014). El diseño de experimentos en la escuela primaria: un diagnóstico de habilidades científicas en niños de 4to grado. Universidad de San Andrés, Buenos Aires, Argentina. 
Elliot, J. (1993). El cambio educativo desde la investigación-acción, Madrid: Morata.

Fensham, (2004). Beyond Knowledge: Other Scientific Qualities as Outcomes for School Science Education. En R.M. Janiuk y E. Samonek-Miciuk (Ed.), Science and Technology Education for a Diverse World-dilemmas, needs and partnerships. International Organization for Science and Technology Education. (IOSTE) XIth Symposium Proceedings (pp. 23-25). Lublin, Polland.

Flórez, R., \& Tobon, A. (2001). Investigación Educativa y Pedagógica. Colombia. McGraw Hill.

García, S., \& Furman, M. (2014). Categorización de preguntas formuladas antes y después de la enseñanza por indagación. Praxis \& Saber, 5(10), 75 91. https://doi.org/10.19053/22160159.3023

Gómez, S., \& Pérez, M. (2013). El pensamiento científico: la incorporación de la indagación guiada a los proyectos de aula. Caldas. Colombia

Garritz, A. (2006). Naturaleza de la ciencia e indagación: cuestiones fundamentales para la educación científica del ciudadano. Revista Iberoamericana de Educación, 42, 127-152.

ICFES. (2015). Lineamientos generales para la presentación de examen de estado Saber 11. Bogotá, D.C.: Mineducacion.

Llewellynd, D. (2005) Teaching High School Science Through Inquiry. Thousand Oaks, CA: Corwin Press.

Libâneo, J.C(1999). Pedagogía e pedagogos, para qué? Saó Paulo. Brasil.

Maggio, M. (2012). Enriquecer la enseñanza. Los ambientes con alta disposición tecnológica como oportunidad. Buenos Aires: Paidós.

Merino de la Fuente, J. M. (2007).Desarrollo curricular de las ciencias experimentales. Granada: Grupo Editorial Universitario.

Muñoz, A. (2014).La indagación como estrategia para favorecer la enseñanza de las ciencias naturales. Palmira. Colombia.

Narváez Burgos, I (2014). La indagación como estrategia en el desarrollo de competencias científicas, mediante la aplicación de una secuencia didáctica en el área de ciencias naturales en grado tercero de básica primaria. Palmira. Colombia.

Ortiz. C (2009). Estrategias didácticas en la enseñanza de las Ciencias Naturales. Revista de Educación \& Pensamiento. Colombia. 
Scheuer, N., Pérez, M., Mateos, M., \& Pozo, I. (2006). Las teorías implícitas sobre el aprendizaje y la enseñanza,. Nuevas formas de pensar la enseñanza y el aprendizaje. pp. 95-132. Barcelona. España.

Restrepo, B. (2004) La investigación-acción educativa y la construcción de saber pedagógico Educación y Educadores, (7), pp. 45-55, Colombia.

Solbes, Montserrat \& Furió (2007). El desinterés del alumnado hacia el aprendizaje de la ciencia: Implicaciones en su enseñanza. Didáctica De Las Ciencias Experimentales y Sociales. N. 21. 2007, 91-117 (ISSN 0214-4379). Departamento de Didáctica de las Ciencias Experimentales y Sociales. Universitad de València.

Solbes \& Vilches, (1997). STS Interactions and the teaching of Physics and Chemistry, Science Education, 81, pp. 377-386.

Tortosa, M. (2012) "The use of microcomputer based laboratories in chemistry secondary education: Present state of the art and ideas for research- based practice.Chem. Educ. Res. Pract..2012, 13, 161-171.

Yager \& Penick, (1986). Perception of four age groups towards science classes, teachers and values of science, Science Education, 70, 353-356. 